\title{
Der Gesellschafterstreit in der radiologischen Berufsausübungsgemeinschaft: Ist Prävention möglich?
}

\section{Der Gesellschafterstreit: ein unerwünschtes Phänomen}

Der Streit zwischen Gesellschaftern einer radiologischen Berufsausübungsgemeinschaft ist ein unerwünschtes Phänomen hierüber sind sich alle Beteiligten rasch einig. Ein solcher Streit kostet nicht nur die Beteiligten Nerven und Geld, sondern wirkt sich auch nachteilig auf Patienten, Mitarbeiter und Reputation der Berufsausübungsgemeinschaft aus. Der Patient argwöhnt, wer streite, konzentriere sich nicht mehr hinreichend auf seine Arbeit; die Mitarbeiter fürchten um ihren Arbeitsplatz.

Das ändert aber nichts daran, dass ein solcher Gesellschafterstreit häufig mit harten Bandagen ausgefochten wird. Das kann an den Charakteren der am Streit beteiligten Gesellschafter liegen, aber auch daran, dass es an einer strukturierten Kommunikation unter den Gesellschaftern mangelt, an insuffizienten oder auslegungsbedürftigen Gesellschaftsverträgen oder daran, dass bei Entwicklung des Gesellschaftsvertrages der Berufsausübungsgemeinschaft die zu berücksichtigenden Interessen entweder nicht artikuliert oder nicht hinreichend ausdiskutiert worden sind.

Dem anwaltlichen Berater, der eine der Streitparteien vertritt oder beauftragt ist, bei der Streitschlichtung behilflich zu sein, stellt sich immer wieder die Frage, ob der Streit im Vorfeld vermeidbar gewesen wäre. Prävention ist nicht nur eine medizinische Kategorie, sondern gleichsam wichtiges Instrument zur Streitvermeidung. Das zeigen die folgenden Beispiele.

\section{Der Streit um die Beteiligung am Vermögen der Berufsaus- übungsgemeinschaft}

Das Bürgerliche Gesetzbuch sieht als Regelfall der Gesellschaft bürgerlichen Rechts vor, dass die Gesellschafter zu gleichen Teilen am Vermögen der Gesellschaft beteiligt sind. Davon kann jedoch durch gesellschaftsvertragliche Regelung abgewichen werden. Wer hier unbedacht zu Werke geht, riskiert Streit.

Zunächst sind die Rahmenbedingungen zu beachten, die durch die Entscheidungen des Bundessozialgerichts (Urteil vom 23.6.2010, Az.: B 6 KA 7/07 R) und des Bundesfinanzhofs (Urteil vom 3.11.2015, Az.: VIII R 62/13) geschaffen worden sind.

Von dem Leitbild der Beteiligung am Vermögen der Gesellschaft zu gleichen Teilen darf nur insoweit abgewichen werden, als nicht ein oder mehrere Gesellschafter ihre Mitunternehmereigenschaft verlieren oder - als verkappte Angestellte - nicht mehr freiberuflich tätig sind. Andernfalls drohen vertragsarztrechtlich unter anderem die Rückforderung der gezahlten Vergütung durch die KV und steuerlich die Gewerblichkeit der Einkünfte.

Der Ausschluss einer Beteiligung am Vermögen der Gesellschaft ist daher nur in engen Grenzen möglich, so z. B. wenn ein Gesellschafter in die Gesellschaft neu aufgenommen wird und die anderen Gesellschafter sich ausbedungen haben, den neu aufgenommenen Gesellschafter bis zum Ablauf einer bestimmten Frist (nach der Rechtsprechung des Bundesgerichtshofs, Urteil vom 7.5.2017, Az.: II ZR 281/05, bis maximal drei Jahre) aus der Gesellschaft hinauskündigen zu können, sollte sich die Zusammenarbeit nicht so gestalten, wie bei Aufnahme erwartet. Der neu aufzunehmende Gesellschafter wird in einem solchen Falle kaum in die Gesellschaft investieren wollen, was die anderen Gesellschafter aber erwarten, wenn der neu aufgenommene Gesellschafter auf einer Vertragsarztzulassung tätig werden soll. Aus diesem Interessenkonflikt kann sich Streit ergeben. Die Beteiligten müssen sich daher - im Wege präventiver Streitvermeidung - darüber klar werden, ob es nicht vorzuziehen ist, dass der neu aufzunehmende Gesellschafter zunächst als Angestellter (und

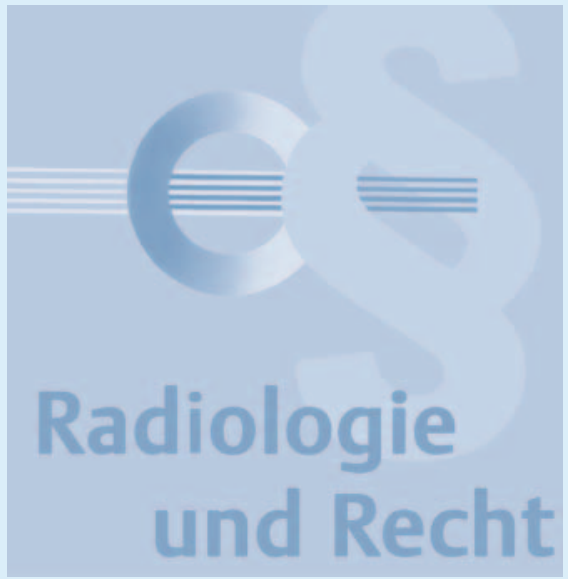

damit ohne Investition) tätig wird und erst nach Ablauf der Kennenlernphase bzw. Probezeit als Gesellschafter aufgenommen wird (unter Übernahme von Vertragsarztzulassung und Gesellschaftsanteilen). In einem solchen Fall konzentriert sich die Vertragsgestaltung auf den Anstellungsvertrag, um in der Übergangsphase Mitwirkungsrechte, aber auch -pflichten zu regeln, die es dem aufzunehmenden Gesellschafter ermöglichen, sich über die „normale“ Tätigkeit als Angestellter hinaus in die Gesellschaft einzubringen, gleichzeitig die anderen Gesellschafter in die Lage zu versetzen, sich ein Bild davon zu machen, ob der aufzunehmende Gesellschafter realistisch als Mitgesellschafter in Betracht kommt.

Streit kann es auch geben, wenn kurz vor dem Ausscheiden eines Gesellschafters eine größere Investition, z. B. Anschaffung eines Großgeräts, durchgeführt werden soll und der ausscheidende Gesellschafter wenig Interesse zeigt, sich an der Investition zu beteiligen.

Eine Regelung - die auf den ersten Blick die Interessen des ausscheidenden und der verbleibenden Gesellschafter berücksichtigt, z. B. wonach ein oder mehrere Gesellschafter berechtigt sind, Sonderbetriebsvermögen zu bilden mit der Folge, dass die anderen Gesellschafter die Investition nicht mitfinanzieren müssen - kann zum Streit führen, wenn es um die Frage geht, wem die Erträge aus der Investition zustehen. Die investierenden Gesellschafter werden (mit einem gewissen Recht) argumentieren, ihnen stünde der Ertrag zu, weil sie 
schließlich investiert und damit finanzielles Risiko getragen hätten; die übrigen Gesellschafter werden (ebenfalls mit einem gewissen Recht) darauf hinweisen, sie könnten von der Verteilung des Ertrags nicht völlig ausgeschlossen werden, weil sie schließlich auch an den Allgemeinkosten, z. B. den Kosten für die Mitarbeiter, die zur Erzielung eines gerätespezifischen Ertrags eingesetzt werden, beteiligt seien. Streitfördernd wäre es, in einem solchen Fall Interessen einseitig durchzusetzen; tragfähiger - weil präventiv streitvermeidend - kann eine Regelung sein, die auf Grundlage einer transparenten Analyse der Anschaffungs- und Folgekosten der Investition sowie der zu erwartenden Erträge entwickelt wird.

Streitbelastet kann weiterhin auch die Verteilung der Anteile an der Berufsausübungsgemeinschaft sein, also die Frage, welcher Gesellschafter in welchem Umfang Gesellschaftsanteile hält.

Beispielsweise kann dies der Fall sein, wenn die Berufsausübungsgemeinschaft einen weiteren Standort übernimmt, der im Verhältnis zur bereits bestehenden Berufsausübungsgemeinschaft einen geringeren $\mathrm{Ge}$ winn erwirtschaftet. Die Bewertung des neuen Standorts wird daher auch einen geringeren Unternehmenswert ergeben mit der Folge, dass der Gesellschaftsanteil des neu aufzunehmenden Gesellschafters niedriger ausfallen wird, als der der anderen Gesellschafter. Einer Regelung, die es dem neu aufzunehmenden Gesellschafter ermöglicht, zum Ausgleich Gesellschaftsanteile hinzuzukaufen, werden die anderen Gesellschafter wegen der damit verbundenen Steuerlast skeptisch gegenüberstehen. Für den neu aufzunehmenden Gesellschafter stellt sich wiederum die Frage, ob er mit seinem - niedrigeren - Gesellschaftsanteil für die Zukunft dauerhaft „eingefroren" sein soll.

Ähnliche Fragen stellen sich, wenn in einer radiologischen Berufsausübungsgemeinschaft einzelne Abteilungen ertragsstärker sind als andere. Dem Wunsch, diesem Ungleichgewicht auch durch eine differenzierte Verteilung der Gesellschaftsanteile Rechnung zu tragen, kann man zunächst entgegenhalten, dass die Berufsausübungsgemeinschaft nach außen als Einheit auftritt und daher der Erfolg der Berufsausübungsgemeinschaft insgesamt letztlich auch auf die ertragsschwächeren Abteilungen gründet. Hilfreich ist auch, sich zu vergegenwärtigen, dass die Bedingungen der Honorarverteilung nicht in Stein gemeißelt sind und die ertragsschwächere Abteilung von heute die ertragsstärkere von morgen sein kann.

Interessenkonflikte dieser Art lassen sich vermeiden, wenn man den Gesellschaftsanteil bei einer Personengesellschaft - ähnlich wie den Kapitalanteil an einer GmbH lediglich als Ausdruck der kapitalmäßigen Beteiligung an der Gesellschaft begreift; das schafft den Raum für die Erkenntnis, dass derartige Fragen leichter über die Gewinn- und Verlustverteilung gelöst werden können.

\section{Der Streit um die Willens- bildung in der Berufsaus- übungsgemeinschaft}

Das Bürgerliche Gesetzbuch sieht als Regelfall vor, dass jeder Gesellschafter eine Stimme hat. Auch von dieser Bestimmung kann durch gesellschaftsvertragliche Regelung abgewichen werden und auch das kann zu Problemen führen.

Eine unterschiedliche Beteiligung am Vermögen der Gesellschaft hat zumeist eine Diskussion zur Folge, ob nicht auch die Stimmen der Gesellschafter nach deren Beteiligung am Vermögen der Gesellschaft gewichtet werden müssten. Hiermit ist zumeist die Aufgabe des Einstimmigkeitsprinzips verbunden, so dass die Gesellschafter mit den geringeren Gesellschaftsanteilen überstimmt werden können. Das kann zu - sich länger hinziehenden und die Berufsausübungsgemeinschaft lähmenden - streitigen Auseinandersetzungen über einzelne Gesellschafterbeschlüsse führen, in deren Zentrum die Frage steht, ob der gesellschaftsvertragliche Minderheitenschutz durch die jeweilige gesellschaftsvertragliche Regelung hinreichend gewahrt ist.

Andererseits kann das Einstimmigkeitsprinzip auch zu einer Blockade der Gesellschaft führen.
Präventiv streitvermeidend wird tätig, wer bei der Entwicklung des Gesellschaftsvertrages oder bei einer Überarbeitung eine Verständigung zwischen den Gesellschaftern herbeiführt, für welche Gesellschafterbeschlüsse unter Berücksichtigung des Minderheitenschutzes welches Quorum gelten soll und mittels Planspiel analysiert, welche Konsequenzen sich für die einzelnen Gesellschafter aus einer solchen - differenzierenden - Regelung ergeben.

\section{Der Streit um die Beteiligung an Gewinn und Verlust der Berufsausübungsgemeinschaft}

Besonders streitanfällig sind Regelungen, die die Gewinn- und Verlustverteilung in einer Berufsausübungsgemeinschaft betreffen. Das Bürgerliche Gesetzbuch sieht als Regelfall vor, dass Gewinn und Verlust zu gleichen Teilen zwischen den Gesellschaftern zu verteilen sind. Davon kann gesellschaftsvertraglich abgewichen werden.

Viele Gesellschaftsverträge sehen vor, dass die Verteilung von Gewinn und Verlust der Beteiligung der Gesellschafter am Vermögen folgt. Derartige Regelungen geraten zumeist dann in die Diskussion, wenn - jüngere - Gesellschafter neu aufgenommen werden sollen, die Sorge haben, dass die verbleibenden - älteren - Gesellschafter in ihrer Arbeitskraft oder Arbeitsbereitschaft nachlassen, ohne dass sich hieraus Auswirkungen auf die Gewinnverteilung ergeben. Die älteren Gesellschafter werden darauf verweisen, dass sie das Unternehmen aufgebaut haben, sie von den jüngeren Gesellschaftern in gleicher Weise Gewinnverzicht zugunsten der älteren Gesellschafter erwarten, wie ihnen dieser auch zugemutet worden ist, oder sie argwöhnen, der neu aufzunehmende jüngere Gesellschafter werde (zunächst) nicht in der gleichen Art und Weise zum Ertrag des Unternehmens beisteuern wie sie selbst.

Diskussionen können auch Regelungen in Gesellschaftsverträgen auslösen, mit denen der Versuch unternommen wird, die Gewinn- und Verlustverteilung zu flexibilisieren und zwar dergestalt, dass Abweichungen vom vereinbarten Arbeitsumfang zu 
einer geringeren bzw. höheren Gewinnund Verlustbeteiligung führen.

Präventiv streitvermeidend wirkt bei derartigen Regelungen die Überlegung, dass die Gewinn- und Verlustbeteiligung nicht nur etwas mit dem Arbeitseinsatz eines Gesellschafters zu tun hat, sondern damit auch sein Kapitaleinsatz (also seine Beteiligung am Vermögen der Gesellschaft) und seine Bereitschaft, mit seinem Privatvermögen zu haften, vergütet wird. Diese Überlegung öffnet den Blick dafür, dass es sinnvoll sein kann, auch bei der Gewinn- und Verlustbeteiligung zu differenzieren, und zwar zwischen einem tätigkeitsbezogenen Gewinn, der ähnlich wie ein Gehalt, nach einem bestimmten Arbeitsumfang (wobei häufig die Frage streitbefangen ist, wie mit verwaltungs- und berufsbezogenen, aber nicht spezifisch praxisbezogenen Tätigkeiten umzugehen ist) bemessen wird, und einem festen - Gehaltsbestandteil, mit dem die Beteiligung am Unternehmen und die Bereitschaft, mit dem Privatvermögen zu haften, vergütet wird.

\section{Der Streit um das Ausscheiden eines Gesellschafters aus der Berufsausübungsgemeinschaft}

Während sich in den bisherigen Beispielen Streitigkeiten noch unter Hinweis auf die weitere Zusammenarbeit der Gesellschafter schlichten lassen, ist das bei Streitigkeiten im Zusammenhang mit dem Ausscheiden eines oder mehrerer Gesellschafter tendenziell nicht der Fall. Das hat seinen Grund darin, dass die verbleibenden Gesellschafter und der oder die ausscheidenden Gesellschafter nicht mehr zusammenarbeiten müssen.

Das Bürgerliche Gesetzbuch sieht für den Fall des Ausscheidens eines Gesellschafters eine einfache Regelung vor: Die Gesellschaft endet. Das ist für eine radiologische Berufsausübungsgemeinschaft, die die Existenz nicht nur der verbleibenden Gesellschafter, sondern auch der Mitarbeiter sichern soll, keine geeignete Lösung. Daher enthalten Gesellschaftsverträge in der Regel sogenannte Fortsetzungsklauseln, also Regelungen, die bewirken, dass die Gesellschaft nach dem Ausscheiden eines Gesellschafters, sei es durch Tod, Berufsunfähigkeit oder Kündigung, von den verbleibenden Gesellschaftern fortgesetzt wird.

Damit beginnen jedoch die Probleme: Mit einer solchen Klausel ist die Rechtsfolge verbunden, dass der Gesellschaftsanteil des ausscheidenden Gesellschafters diesem ab- und den verbleibenden Gesellschaftern anwächst.

Das wirft aus der Sicht der verbleibenden Gesellschafter die Frage auf, was mit der Zulassung des ausscheidenden Gesellschafters geschieht, die von dem Rechtsvorgang der Ab- und Anwachsung des Gesellschaftsanteils nicht umfasst wird. Soll die Zulassung an die Gesellschaft gebunden werden (ähnlich wie bei einem MVZ) und wie rechtssicher ist eine solche Regelung (es gibt Gerichtsentscheidungen, so LG Dortmund, Urteil vom 27.9.2007, Az.: 3 0391/07, die eine solche Regelung bestätigt haben, aber keine höchstrichterliche Entscheidung) oder soll der ausscheidende Gesellschafter frei über seine Zulassung verfügen können, was aber den Fortbestand des Unternehmens schwächt und Auswirkungen auf die Abfindung des ausscheidenden Gesellschafters haben muss? Oder soll sich der ausscheidende Gesellschafter selbst um die Verwertung seines Gesellschaftsanteils und seiner Zulassung kümmern, was z. B. bei überörtlichen Berufsausübungsgemeinschaften mit mehreren Standorten in Betracht kommt und auf eine Realteilung hinausläuft? Das hat weitere Fragen zur Folge, z. B. welche Reichweite in einem solchen Fall ein Wettbewerbsverbot für den ausscheidenden Gesellschafter haben kann und muss.

Aus Sicht des ausscheidenden Gesellschafters ist z. B. klärungsbedürftig, wie er einem Streit um die Höhe seines Abfindungsanspruchs entgehen kann.

Dass es um das Ausscheiden eines Gesellschafters häufig Streit gibt, liegt (neben der Tatsache, dass im Zusammenhang mit der Gestaltung der Ausscheidensregelungen mehrere Rechtsmaterien zusammenwirken) auch daran, dass den Ausscheidensregelungen bei der Erarbeitung des Gesellschaftsvertrages nicht hinreichend Aufmerksamkeit geschenkt wird. Hier wirken ähnliche Mechanismen wie bei der Befassung mit Themen der Altersvorsorge.
Das Ausscheiden eines Gesellschafters ist ein Sachverhalt, der bei der Gründung einer Gesellschaft nicht im Fokus steht; kommt es zum Ausscheiden eines Gesellschafters, so sind nachträgliche Gestaltungen zumeist wegen der diametral entgegengesetzten Interessen nicht mehr möglich.

Präventiv streitvermeidend wirken daher die Gesellschafter, die - wie bei einem Planspiel - die möglichen Alternativen für ein Ausscheiden und die sich hieraus ergebenden Rechtsfolgen durchspielen und auf Grund einer solchen Analyse eine möglichst die Interessen aller Gesellschafter berücksichtigende Regelung erarbeiten.

\section{Voraussetzungen präventiver Streitvermeidung}

Wie bei der Prävention in der Gesundheitsvorsorge setzt auch eine erfolgreiche Streitvermeidung die Bereitschaft voraus, sich auf präventive Maßnahmen einzulassen.

Der Erfolg präventiver Bemühungen ist schwer messbar, da alternative Geschehensabläufe als Vergleichsmaßstab fehlen. Intensität und Dauer eines vermiedenen Gesellschafterstreits lassen sich nur abstrakt beschreiben, entsprechend lassen sich die Kosten der Streitvermeidung sich nicht in Relation zu den Kosten eines vermiedenen Gesellschafterstreits setzen. Dies reduziert die Bereitschaft für präventive Maßnahmen; zum Ausdruck kommt ein solches Verhalten in dem Rückgriff auf „bewährte“ Vertragsmuster (ohne deren Anwendbarkeit auf die spezifischen Belange der Gesellschafter zu hinterfragen) oder in dem Bemerken, den Gesellschaftsvertrag brauche man ja nur für den Zulassungsausschuss, danach verschwinde er in der Schublade.

Neben einer grundsätzlichen Bereitschaft, sich auf präventive Maßnahmen einzulassen, gibt es weitere Voraussetzungen für eine erfolgreiche Streitvermeidung:

Hierzu zählen zunächst Offenheit und Transparenz, d. h. die Bereitschaft, die Diskussion auch unangenehmer Themen zuzulassen und dabei jeden anderen Gesellschafter ernst zu nehmen. Hierzu gehört auch die Bereitschaft zur Analyse strittiger 
Sachverhalte, ggfs. unter Hinzuziehung externer Expertise.

Eine weitere Voraussetzung für eine erfolgreiche Streitvermeidung ist es, sich Zeit für die Diskussion komplexer Sachverhalte zu nehmen, aus denen sich später Streitfälle ergeben können. Hilfreich ist, mit der Erarbeitung von Gesellschaftsverträgen, die häufig wegen anstehender Termine beim Zulassungsausschuss unter Zeitdruck stehen, rechtzeitig zu beginnen und diese Phase als eine Art Training für etwaige Streitfälle zu betrachten. Der Gesellschaftsvertrag ist ein wesentliches Instrument der Streitvermeidung. Er regelt - oder sollte es zumindest - in einer Berufsausübungsgemeinschaft typischerweise entstehende Konflikte. Die Phase der Erarbeitung des Gesellschaftsvertrages bietet also eine gute Möglichkeit, im Wege der Diskussion der in Betracht kommenden Lösungen für diese typischerweise entstehenden Konflikte Lösungsalternativen durchzuspielen und Streitkultur einzuüben oder rechtzeitig zu erkennen, dass die Basis für eine erfolgreiche Zusammenarbeit zwischen den Gesellschaftern fehlt - der beste Schutz vor dem „querulatorischen“ Gesellschafter.

Hilfreich ist auch, die Gründe für bestimmte gesellschaftsvertragliche Lösungen schriftlich niederzulegen, damit darauf - erinnernd - im Streitfalle mit ggfs. streiteindämmender Wirkung zurückgegriffen werden kann.
Alles verändert sich, auch die Rahmenbedingungen, die bei Bearbeitung eines Gesellschaftsvertrages zugrunde gelegt worden sind. Es dient also auch der Prävention, Gesellschaftsverträge in angemessenen zeitlichen Abständen oder wenn sich wesentliche Rahmenbedingungen ändern, einer Prüfung dahingehend zu unterziehen, ob sie noch ausreichend die Belange der Gesellschafter abbilden oder veraltete Regelungen möglicherweise den Streit von morgen verursachen können.

\section{Neues Personengesell- schaftsrecht}

Eine gute Gelegenheit, Gesellschaftsverträge einer Überprüfung zu unterziehen, bietet die vom Gesetzgeber geplante Neufassung des Personengesellschaftsrechts, für die das Bundesjustizministerium im April 2020 den Vorschlag (Gesetz zur Modernisierung des Personengesellschaftsrechts, MOPEG) einer Expertenkommission vorgelegt hat. Da das Personengesellschaftsrecht maßgebliche Grundlage der in der Rechtsform der Gesellschaft bürgerlichen Rechts oder der Partnerschaftsgesellschaft betriebenen Berufsausübungsgemeinschaft ist, werden die geplanten Änderungen Auswirkungen auch auf die radiologischen Berufsausübungsgemeinschaften haben. Hier ein kurzer Ausblick auf einige der geplanten Änderungen:
- Rechtsfähigkeit der Gesellschaft bürgerlichen Rechts (GbR); sie ist rechtsfähig, „wenn sie nach dem gemeinsamen Willen der Gesellschafter am Rechtsverkehr teilnehmen soll“.

- Freiwillige Eintragung in das neu zu schaffende Gesellschaftsregister („eGBR“); die Eintragung schafft unter anderem Namensschutz für die eGBR.

- Die Anfechtung von Gesellschafterbeschlüssen der GbR wird nach aktienrechtlichem Vorbild gestaltet, d. h. es muss eine fristgebundene Anfechtungsklage erhoben werden.

- Personenverbindungen freier Berufe können sich in das Handelsregister eintragen lassen, was den Zugang zur Rechtsform der GmbH \& Co KG ermöglicht; allerdings nur, wenn das Berufsrecht das zulässt.

- Weitere Änderungen betreffen das Ausscheiden eines GbR-Gesellschafters (keine Auflösung der Gesellschaft als Regelfall mehr).

Wann das Gesetzesvorhaben umgesetzt wird, ist noch nicht abzusehen, derzeit laufen die Anhörungen. Wir werden berichten.

Dr. Horst Bonvie

Rechtsanwalt

Fachanwalt für Medizinrecht

Rechtsanwälte Wigge

Großer Burstah 42

20457 Hamburg 